\title{
Distorção da imagem corporal em bailarinas jovens
}

\section{Body image distortion in young dancers}

\section{Distorción de imagen corporal en balerina joven}

\author{
(iD) Iris lasmine de Rezende Araújo \\ Universidade Estadual de Goiás (UEG), Anápolis, Goiás, Brasil \\ E-mail: irisiasmine@hotmail.com \\ iD (9) Lucas Henrique Ferreira Sampaio \\ Universidade Estadual de Goiás (UEG), Goiânia, Goiás, Brasil \\ E-mail: lucas.sampaio@ueg.br \\ iD 9 Adriano Jabur Bittar \\ Universidade Estadual de Goiás (UEG), Goiânia, Goiás, Brasil \\ E-mail: adriano@studioabittar.com \\ iD Tânia Cristina Dias da Silva Hamu \\ Universidade Estadual de Goiás (UEG), Goiânia, Goiás, Brasil \\ E-mail: tania.ft@gmail.com \\ (iD) Leonardo Alves Rezende \\ Universidade Estadual de Goiás (UEG), Goiânia, Goiás, Brasil \\ E-mail: leo_alves182@hotmail.com \\ (D) Cibelle Kayenne Martins Roberto Formiga \\ Universidade Estadual de Goiás (UEG), Goiânia, Goiás, Brasil \\ E-mail: cibellekayenne@gmail.com
}

Resumo: O objetivo do estudo foi analisar a imagem corporal de bailarinas jovens e relacionar com suas medidas antropométricas. Um objetivo secundário foi comparar as medidas antropométricas aferidas e referidas pelas bailarinas. O estudo teve delineamento transversal com uma amostra de 33 bailarinas entre 13 e 17 anos de idade. Foram coletados dados biossocioeconômicos, e a imagem corporal foi avaliada através do 
Body Shape Questionnaire (BSQ), para determinar a sua distorção e preocupação. Os resultados mostraram que a maioria das bailarinas apresentou distorção da imagem corporal, no entanto, houve baixa prevalência de preocupação com a imagem corporal. Além disso, as bailarinas subestimam seu índice de massa corporal em relação à medida aferida pelos pesquisadores.

Palavras-chave: dança; imagem corporal; hábitos.

Abstract: The aim of the study was to analyze body image and relate it to anthropometric measurements of young dancers. The secondary objective was to compare the anthropometric measures measured and reported by the dancers. The study had a cross-sectional design with a sample of 33 dancers between 13 and 17 years of age. Socioeconomic data were collected, and body image was assessed using the Body Shape Questionnaire (BSQ) to determine distortion and concern with body image. The results showed that most dancers presented distortion of body image, however there was a low prevalence of concern with body image. In addition, the dancers underestimate their body mass index in relation to the measurement measured by the researchers.

Keywords: dance; body image; habits.

Resumen: El objetivo del estudio fue analizar la imagen corporal y relacionarla con mediciones antropométricas de jóvenes bailarines. El objetivo secundario fue comparar las medidas antropométricas medidas e informadas por los bailarines. El estudio tuvo un diseño transversal con una muestra de 33 bailarines entre 13 y 17 años. Se recogieron datos biosocioeconómicos y se evaluó la imagen corporal utilizando el cuestionario de forma corporal (BSQ) para determinar la distorsión y la preocupación por la imagen corporal. Los resultados mostraron que la mayoría de los bailarines presentaron distorsión de la imagen corporal, sin embargo, hubo una baja prevalencia de preocupación con la imagen corporal. Además, los bailarines subestiman su índice de masa corporal en relación con la medición medida por los investigadores.

Palabras Ilave: danza; imagen corporal; hábitos

Submetido em: 13-12-2019

Aceito em: 23-06-2020 


\section{Introdução}

A imagem corporal é um construto multidimensional que envolve aspectos psicológicos, fisiológicos e sociológicos, podendo ser definida como a representação mental do próprio corpo (SCHILDER, 1981; THOMPSON, 1990). Aspectos comportamentais, cognitivos, afetivos e perceptuais provenientes das experiências corporais vivenciadas estão diretamente relacionadas à imagem corporal (CASH; PRUZINSKY, 2002). Pode ser influenciada por fatores biológicos (peso, idade e sexo), socioeconômicos (escolaridade, renda, classe econômica e local de moradia) e características psicológicas (internalização, autoestima e perfeccionismo). Uma imagem corporal negativa pode surgir durante a infância e é um fator de risco para o desenvolvimento de psicopatologias futuras (FELDEN et al., 2015; NEVES et al., 2017; NICHOLS et al., 2018).

O balé clássico é uma forma de dança ensinada no mundo inteiro e sua rotina exige ensaios com ênfase na sustentação, equilíbrio, sapatilhas de ponta e um estereótipo de corpo magro elegante (HAAS et al., 2010). A procura de um corpo ideal entre as bailarinas promove substanciais alterações da composição corporal; em geral, elas têm menor massa corporal e são mais flexíveis que a média das mulheres da população (DOURADO et al., 2012). Pesquisas apontam que bailarinas têm maior risco de desenvolver deficiências nutricionais e distorção da imagem corporal (MOURA et al., 2015; CASTRO; MAGAJEWSKI; LIN, 2017). A partir do momento em que bailarinos se tornam profissionais, aumenta a exigência da manutenção de um padrão estético com baixo peso. Deste modo, a alimentação constitui um fator imprescindível para o desenvolvimento das características necessárias a essa modalidade artística, por influenciar na composição corporal, na aptidão física, na prevenção de lesões e no maior desempenho por parte dos praticantes (MOURA et al., 2015).

No entanto, apesar dos bailarinos apresentarem composição corporal e características antropométricas eutróficas, é comum o 
desejo por um corpo mais magro (CARDOSO, 2017). As distorções e anormalidades da imagem corporal podem alterar a maneira como bailarinos codificam seu corpo, podendo influenciar na ocorrência de distúrbios em sua saúde física e mental (ZIKAN, 2019).

O período da adolescência é considerado muito significativo para a estruturação da imagem corporal (CONTI, 2008). Saúde, estética e autoestima são os principais fatores que influenciam a imagem corporal em adolescentes (PETROSKI; PELEGRINI; GLANER, 2012), assim como a associação entre sexo e composição corporal (FELDEN et al., 2015). Considerando que os adolescentes passam por diversas transformações corporais que podem ser influenciadas positiva ou negativamente pelo ambiente em que vivem, torna-se importante investigar a forma como percebem a sua imagem corporal dentro do contexto da dança.

A avaliação da imagem corporal desperta interesse de profissionais e pesquisadores, sendo a mesma avaliada sob a ótica de fatores como percepção da própria imagem corporal, cognição e afeto sobre o próprio corpo e comportamentos e atitudes alimentares (BANFIELD; MCCABE, 2002).

Alguns instrumentos têm sido usados rotineiramente para avaliação da imagem corporal em crianças, adolescentes e adultos. O Body Shape Questionnaire (BSQ) é um instrumento amplamente utilizado para avaliação da imagem corporal. Embora tenha sido desenvolvido originalmente para avaliar a preocupação sobre as formas do corpo em pacientes com distúrbios alimentares (COOPER et al., 1987), tem sido utilizado também para avaliar a satisfação com a imagem corporal (LAPORTA-HERRERO et al., 2018), preocupação com a imagem corporal (DI PIETRO; SILVEIRA, 2009) e distorção da imagem corporal (HAAS; GARCIA; BERTOLETTI, 2010) em diversas populações. Além do uso de instrumentos, a imagem corporal também tem sido avaliada por meio de pesquisas qualitativas, mediante entrevistas realizadas com crianças ou com seus cuidadores (CRUZ et al., 2020). 
Neste sentido, o objetivo do estudo foi analisar a imagem corporal de bailarinas jovens e relacionar com suas medidas antropométricas. Um objetivo secundário do estudo foi comparar as medidas antropométricas aferidas e referidas pelas bailarinas.

\section{Materiais e Métodos}

\section{Amostra e delineamento do estudo}

Estudo de delineamento observacional transversal analítico prospectivo, com amostra composta por 33 bailarinas semiprofissionais recrutadas no Centro de Artes Basileu França, em Goiânia-Goiás. A amostra elegível foi composta por 42 bailarinas, mas apenas 33 participantes finalizaram todas as avaliações propostas nesta pesquisa. As coletas foram realizadas no Laboratório de Pesquisa em Musculoesquelética (LAPEME) da Universidade Estadual de Goiás (UEG). Critérios de inclusão: ter idade menor que 18 anos, tempo de dança superior a quatro anos, não estar afastada dos ensaios por motivo de lesão ou doenças.

\section{Aspectos éticos}

A pesquisa foi aprovada pelo Comitê de Ética e Pesquisa do Hospital das Clínicas da Universidade Federal de Goiás (UFG), CAAE: 64312216.8.0000.5078. O consentimento foi obtido por meio do Termo de Assentimento Livre e Esclarecido (TALE) e Termo de Consentimento Livre e Esclarecido (TCLE) para as participantes e seus responsáveis, respectivamente.

\section{Coleta de dados}

Foram coletados os dados de identificação das bailarinas (idade, escolaridade, endereço, telefone, profissão), idade de início da prática da dança, tempo de dança, duração diária de treinos 
realizados, peso e altura referidos pela bailarina e condições de saúde atuais e pregressas por meio de um questionário de anamnese. Foi aplicado também o Questionário ABEP 2015 (Associação Brasileira de Empresas de Pesquisa) para classificar e caracterizar a condição econômica das famílias (nível A1, A2, B1, B2, C, D e E).

Foram avaliados os dados antropométricos de peso, altura, índice de massa corporal (IMC), dobras cutâneas, circunferências e medidas antropométricas pelo protocolo da International Society for Advancement of Kinanthropometry (ISAK) (LOPES; RIBEIRO, 2013).

\section{Avaliação da Imagem Corporal}

A imagem corporal foi investigada através do Questionário Body Shape Questionnaire (BSQ), uma ferramenta autoaplicativa composta por 34 questões com seis opções de reposta (nunca $=1$, raramente $=2$, às vezes $=3$, frequentemente $=4$, muito frequentemente $=5$ e sempre $=6$ ). A pontuação no questionário fica entre 34 e 204 pontos.

A distorção da imagem corporal foi determinada através do escore obtido no BSQ e classificados como normal $(<80)$, leve distorção (81 - 110), moderada distorção (111 - 140), e grave distorção (>140) (HAAS; GARCIA; BERTOLETTI, 2010); e a preocupação com a imagem corporal determinada também através do escore obtido no BSQ e classificados como nenhuma $(<110)$, preocupação leve (111-138), preocupação moderada (139-167), e preocupação grave (>168) (DI PIETRO; SILVEIRA, 2009).

\section{Análise dos dados}

Os dados foram analisados em duas etapas. A normalidade dos dados foi verificada por meio do teste Kolmogorov-Smirnov. A análise estatística descritiva dos dados foi processada utilizando-se média e desvio-padrão para as variáveis contínuas, e cálculo de frequência e porcentagem para as variáveis discretas ou categóri- 
Distorção da imagem corporal em bailarinas jovens

Iris lasmine de Rezende Araújo - Lucas Henrique Ferreira Sampaio • Adriano Jabur Bittar • Tânia Cristina Dias da Silva Hamu • Leonardo Alves Rezende • Cibelle Kayenne Martins Roberto Formiga

cas. O teste de correlação de Spearmann foi utilizado com o objetivo de verificar a correlação entre a distorção da imagem corporal (BSQ) e as variáveis antropométricas. O teste T para amostras pareadas foi utilizado com o objetivo de comparar as médias das variáveis antropométricas (peso, altura e IMC) aferidas e referidas pelas bailarinas. A análise estatística foi realizada com o programa estatístico Statistical Package for Social Sciences (SPSS), versão 23.0. Foi considerado o nível de significância estatístico de $5 \%(p<0,05)$ em todas as análises.

\section{Resultados}

Foram avaliadas 33 bailarinas com idade entre 13 e 17 anos, sendo que as características coletadas por meio de questionário de anamnese encontram-se apresentadas na Tabela 1:

Tabela 1: Características dos bailarinos da amostra $(n=33)$

\begin{tabular}{lc}
\hline Características dos bailarinos & Valores \\
\hline Idade (anos) - Média (DP) & $14,6( \pm 1,2)$ \\
Peso Corporal (kg) - Média (DP) & $49,09( \pm 7,2)$ \\
Altura (cm) - Média (DP) & $123( \pm 0,6)$ \\
Índice de Massa Corporal - IMC (kg/m²) - Média (DP) & $19,1( \pm 1,83)$ \\
Classificação do IMC - f (\%) & \\
$\quad$ Abaixo do peso & $3(10)$ \\
$\quad$ Eutrófica & $30(90)$ \\
Quantidade de tempo em anos que praticam a dança - Média (DP) & $8,4( \pm 2,0)$ \\
Média de horas de aula/ensaios por semana - Média (DP) & $4,3( \pm 1,9)$ \\
Classificação Econômica (ABEP) & \\
Classe A - $f(\%)$ & $9(27,3)$ \\
Classe B1 - $f(\%)$ & $11(33,3)$ \\
Classe B2 - $f(\%)$ & $6(18,2)$ \\
Classe C 1 - $f(\%)$ & $5(15,2)$ \\
Classe C2 - $f(\%)$ & $1(3,0)$ \\
Classe D - E - $f(\%)$ & $1(3,0)$ \\
\hline
\end{tabular}

Nota: f - frequência; \% - porcentagem; DP - desvio padrão; Mín - valor mínimo; Máx - valor máximo; ABEP Associação Brasileira de Empresas e Pesquisa.

Fonte: elaboração própria. 
Distorção da imagem corporal em bailarinas jovens

Iris lasmine de Rezende Araújo - Lucas Henrique Ferreira Sampaio • Adriano Jabur Bittar • Tânia Cristina Dias da Silva Hamu • Leonardo Alves Rezende • Cibelle Kayenne Martins Roberto Formiga

A Tabela 2 refere-se aos hábitos e problemas de saúde pré-existentes na amostra. Verifica-se que os problemas de saúde mais frequentes das bailarinas foram: doença do trato gastrointestinal, lesões articulares, doença respiratória e desmaios. Uma minoria das bailarinas relatou hábitos prejudiciais à saúde para emagrecer.

Tabela 2: Características das condições de saúde da amostra ( $n=33)$

\begin{tabular}{lcc}
\hline Problemas de saúde & $\mathbf{f}$ & $\%$ \\
Doença respiratória & 7 & 21,2 \\
Desmaios & 7 & 21,2 \\
Convulsões & 1 & 3,0 \\
Diabetes & 1 & 3,0 \\
Doença do trato gastrointestinal & 8 & 24,2 \\
Anemia & 3 & 9,1 \\
Doenças Renais & 1 & 3,0 \\
Lesão articular prévia & 8 & 24,2 \\
\hline Hábitos prejudiciais à saúde para emagrecer & $\mathbf{f}$ & $\%$ \\
\hline Induziu vômito & 5 & 15,2 \\
Utilizou laxante & 5 & 15,2 \\
\hline
\end{tabular}

Os resultados da avaliação da imagem corporal estão expressos na Tabela 3. É possível observar que a maioria das bailarinas apresenta distorção da imagem corporal de leve a grave e, apesar disso, uma minoria demonstrou ter preocupação com a imagem corporal. 
Distorção da imagem corporal em bailarinas jovens

Iris lasmine de Rezende Araújo - Lucas Henrique Ferreira Sampaio • Adriano Jabur Bittar • Tânia Cristina Dias da Silva Hamu • Leonardo Alves Rezende • Cibelle Kayenne Martins Roberto Formiga

Tabela 3: Resultados da avaliação da imagem corporal $(n=33)$

\begin{tabular}{lcc}
\hline Distorção da Imagem Corporal & f & $\%$ \\
Não apresenta & 16 & 48,5 \\
Leve distorção & 10 & 30,3 \\
Moderada distorção & 4 & 12,1 \\
Grave distorção & 3 & 9,1 \\
Preocupação com a Imagem Corporal & $\mathbf{f}$ & $\%$ \\
Nenhuma preocupação & 26 & 78,8 \\
Preocupação leve & 4 & 12,1 \\
Preocupação moderada & 2 & 6,1 \\
Preocupação grave & 1 & 3,0 \\
\hline \multicolumn{2}{c}{ Nota: f-frequência; \% - porcentagem } \\
$\quad$ Fonte: elaboração própria.
\end{tabular}

A Tabela 4 apresenta os resultados da relação entre os dados antropométricos referidos pelos participantes com a presença de distorção da imagem corporal.

Tabela 4: Correlação entre a distorção da imagem corporal e medidas antropométricas $(n=33)$

\begin{tabular}{lcc}
\hline Distorção da imagem corporal & $\begin{array}{c}\text { Coeficiente de } \\
\text { correlação de } \\
\text { Spearman }\end{array}$ & $\mathbf{p}$ \\
\hline Peso referido & 0,74 & $<0,001$ \\
Altura referida & 0,36 & 0,043 \\
IMC referido & 0,74 & $<0,001$ \\
Circunferência do quadril (aferido) & 0,68 & $<0,001$ \\
\hline
\end{tabular}

Nota: IMC - índice de massa corporal; p - valor de p ou nível de significância. Fonte: elaboração própria.

Na Tabela 5 é possível observar que as bailarinas referem peso mais baixo e maior altura do que os valores aferidos através da avaliação antropométrica. Contudo, os resultados foram estatisticamente significativos apenas para o IMC aferido e referido. 
Tabela 5: Comparação de médias entre medidas antropométricas aferidas e referidas $(n=33)$

\begin{tabular}{ccccc}
\hline Variáveis & $\begin{array}{c}\text { Dados aferidos } \\
\text { Média (DP) }\end{array}$ & $\begin{array}{c}\text { Dados referidos } \\
\text { Média (DP) }\end{array}$ & $\begin{array}{c}\text { Intervalo de Confian- } \\
\text { ça } \\
\mathbf{9 5 \%}\end{array}$ & p \\
\hline Peso (kg) & $50,7( \pm 1,26)$ & $49,0( \pm 1,49)$ & $(-3,760-0,360)$ & 0,101 \\
Altura (cm) & $161( \pm 0,01)$ & $162( \pm 0,01)$ & $(-0,00094-0,015)$ & 0,081 \\
IMC $\left(\mathbf{k g} / \mathbf{m}^{2}\right)$ & $19,4( \pm 0,41)$ & $18,5( \pm 0,42)$ & $(-1,67--0,046)$ & 0,039 \\
\hline
\end{tabular}

Nota: IMC - indice de massa corporal; DP - desvio padrão; $p$ - valor de p ou nível de significância. Fonte: elaboração própria.

\section{Discussão}

Este estudo analisou a relação entre a imagem corporal e características antropométricas de bailarinas jovens. Os achados mostram uma prevalência de meninas magras e de classes econômicas privilegiadas. $\mathrm{Na}$ análise das medidas antropométricas do estudo identificou-se que a maioria das bailarinas avaliadas apresentaram relação peso/altura (IMC) adequada de acordo com a Organização Mundial de Saúde (2006).

Estes achados corroboram um estudo realizado com bailarinas neozelandesas que apresentaram resultados semelhantes, com IMC médio de 19,7 kg/m² e em sua maioria com peso saudável para a idade (BECK et al., 2015). Um estudo recente realizado com bailarinos espanhóis também observou uma média de IMC semelhante $(19,7 \mathrm{~kg} / \mathrm{m} 2)$, no entanto os bailarinos clássicos e contemporâneos apresentaram distorção da imagem corporal percebendo-se maiores do que realmente são (VAQUERO-CRISTÓBAL; KAZAREZ; ESPARZA-ROS, 2017).

De acordo com os dados levantados na anamnese, os problemas de saúde mais frequentes das bailarinas foram as doenças do trato gastrointestinal, lesões articulares e problemas respiratórias. Estudo aponta que de 20 a 50\% da população praticante de esportes de longa duração apresenta pelo menos um sintoma como, por exemplo, vômitos, náuseas, pirose retroesternal (azia), diar- 
reia, cólica abdominal, perda de apetite e sangramento (REIS et al., 2013). Apesar de uma minoria de bailarinas possuir hábitos de uso de laxantes e indução de vômitos para emagrecer, não é possível estabelecer uma relação causal entre esses hábitos e os problemas gastrointestinais observados (BEESE, 2019). Neste sentido, há necessidade de maior investigação desse aspecto para o melhor acompanhamento de saúde geral dessas bailarinas. Vale salientar que no atual estudo os hábitos alimentares não foram o alvo de investigação e as medidas de saúde foram coletadas baseadas em informações reportadas em questionários com as participantes.

Neste estudo, a maioria das bailarinas apresenta distorção da imagem corporal, porém a minoria apresentou sinais de preocupação com a imagem corporal. Os bailarinos vivenciam exigências, expectativas e pressões por parte das companhias de dança, dos colegas, da plateia e acabam sendo corporificados (HAAS; GARCIA; BERTOLETTI, 2010). Sustentando a corporificação como parte de seu trabalho, os dados aqui encontrados corroboram os de outros estudos realizados com bailarinos, evidenciando um padrão de corpo magro, jovem, esbelto, com braços e pernas compridos, quadris e ombros com a mesma proporção (KUMAR, 2001; MONTEIRO; CORREA, 2013).

O culto à magreza e a busca pelo perfeccionismo geram uma pressão constante sobre os bailarinos e os tornam um grupo de risco importante para transtornos de imagem corporal mais graves, quando comparados com outros grupos da mesma idade (CASTRO; MAGAJEWSKI; LIN, 2017; MURNEN; SMOLAK, 2019). Neste sentido, a imagem corporal idealizada para os padrões da dança pode afetar a forma que a bailarina, especialmente na fase da adolescência, percebe o próprio corpo junto aos seus pares. As meninas adolescentes podem apresentar mais problemas com a imagem corporal devido a diversos fatores associados, tais como o nível de atividade física e as informações veiculadas na mídia (AÑEZ et al., 2018).

Neste estudo, observou-se diferença entre as medidas antropométricas aferidas pelos pesquisadores e referidas pelas baila- 
rinas, contudo as diferenças foram estatisticamente significativas apenas para o cálculo do IMC. A subestimação de medidas é um importante indicador da presença de distorção da imagem corporal. Como a amostra foi composta por meninas de 13 a 17 anos, acredita-se que o resultado pode estar relacionado à imaturidade e baixo nível de reconhecimento das mudanças corporais nessa faixa etária. Bailarinos mais jovens tendem a apresentar distorção da imagem corporal, acreditando ter um IMC maior do que é realmente, enquanto os bailarinos mais velhos apresentam melhor autopercepção sobre suas características corporais (KAZAREZ; VAQUERO-CRISTÓBAL; ESPARZA-ROS, 2018).

Um estudo recente realizado com adolescentes brasileiros observou uma alta prevalência de distorção da imagem corporal, sendo a subestimação da imagem corporal prevalente naqueles com sobrepeso e obesos e a superestimação da imagem corporal observadas em adolescentes eutróficos (NOGUEIRA-DE-ALMEIDA et al., 2018). A associação entre sexo feminino e maior IMC é considerada fator crucial para distorção da imagem corporal em adolescentes (FERNÁNDEZ-BUSTOS et al., 2019).

Compreender a percepção dos dançarinos sobre os fatores que influenciam sua insatisfação corporal em contextos de dança é importante para subsidiar programas de prevenção que incentivem a valorização positiva da imagem corporal e hábitos alimentares saudáveis nas escolas de dança (DANTAS et al., 2018).

O presente estudo apresenta como limitações o tamanho amostral, informações de saúde baseadas em autorrelato e a coleta de medidas antropométricas simples. Sugere-se que em estudos futuros sejam coletados dados de composição corporal através de bioimpedância para determinar outras medidas, como percentual de gordura, densidade óssea e massa muscular. Apesar disso, os resultados apresentam importantes desdobramentos para os estudos de imagem corporal e o balé profissional, podendo contribuir para o desenvolvimento da abordagem da equipe multiprofissional com esta população. Mesmo com as limitações citadas, o estudo cumpriu os objetivos e traz o alerta sobre a atenção e o 
cuidado que os profissionais da dança devem ter em relação aos aspectos estudados.

\section{Conclusão}

O estudo concluiu que a maioria das bailarinas jovens estudadas apresentaram distorção da imagem corporal e este resultado teve correlação diretamente proporcional com as medidas antropométricas referidas e aferidas. Além disso, as bailarinas subestimam seu índice de massa corporal em relação à medida aferida pelos pesquisadores. Ressalta-se que os achados deste estudo contemplam um contexto específico de uma amostra regional, não devendo ser extrapolados para todos os contextos.

\section{Referências}

AÑEZ, Elizabeth et al. Body image dissatisfaction, physical activity and screen-time in Spanish adolescents. Journal of Health Psychology, Londres, v. 23, n. 1, p. 36-47, 2018.

BANFIELD Sophie; MCCABE Marita. An evaluation of the construct of body image. Adolescence, San Diego, v. 37, n. 146, p. 373-393, 2002.

BECK, Kathryn L. et al. Dietary intake, anthropometric characteristics, and iron and vitamin $D$ status of female adolescent ballet dancers living in New Zealand. International journal of sport nutrition and exercise metabolism, Bath, v. 25, n. 4, p. 335-343, 2015.

BEESE, Sophie Elizabeth et al. Body image dissatisfaction in patients with inflammatory bowel disease: a systematic review. BMJ open gastroenterology, Birmingham, v. 6, n. 1, p. 1-16, 2019. 
CARDOSO, Allana Alexandre et al. Study of Body Image in Professional Dancers: a Systematic Review. Revista Brasileira de Medicina do Esporte, Vila Mariana, v. 23, n. 4, p. 335-340, 2017. CASH, Thomas; PRUZINSKY, Thomas (orgs). Body Image: a handbook of theory, research and clinical practive. New York: Guilford Press, 530 p., 2002.

CASTRO, Caroline Borges; MAGAJEWSKI, Flávio; LIN, Jaime. Atitudes alimentares e autopercepção da imagem corporal em bailarinas do município de Tubarão-Santa Catarina. Arquivos Catarinenses de Medicina, Santa Catarina, v. 46, n. 1, p. 33-42, 2017.

COOPER, Peter J. et al. The development and validation of the Body Shape Questionnaire. International Journal of eating disorders, Denver, v. 6, n. 4, p. 485-494, 1987.

CONTI, Maria Aparecida. Os Aspectos que Compõem o Conceito de Imagem Corporal pela Ótica do Adolescente. Revista Brasileira de Crescimento e Desenvolvimento Humano, Santo André, v. 18, n. 3, p. 240-253, 2008.

CRUZ, Letícia Maria Cunha et al. Imagem corporal e sexualidade na infância: uma abordagem qualitativa. Pensar a prática, Goiânia, v. 23, n. 1, p. 1-23, 2018.

DANTAS, Ana Garcia et al. Factors Dancers Associate with their Body Dissatisfaction. Body Image, Ohio, v. 25, p. 40-47, 2018.

DI PIETRO, Monica; SILVEIRA, Dartiu Xavier da. Validade interna, dimensionalidade e desempenho da escala Body Shape Questionnaire em uma população de estudantes universitários brasileiros. Revista Brasileira de Psiquiatria, Rio de Janeiro, v. 31, n. 1, p. 21-24, 2009.

DOURADO, Camila Pereira et al. Perfil nutricional de adolescentes praticantes de balé clássico do município de Guarapuava/ Paraná. Revista Brasileira de Nutrição Esportiva, São Paulo, v. 6, n. 35, p. 9, 2012. 
FELDEN, Érico Pereira Gomes et al. Fatores sociodemográficos e imagem corporal em adolescentes do ensino médio. Ciência \& Saúde Coletiva, Rio de Janeiro, v. 20, n. 11, p. 3329-3337, 2015. FERNÁNDEZ-BUSTOS, Juan Gregorio et al. Body Dissatisfaction in Adolescents: Diferences by Sex, BMI and Type and Organisation of Physical Activity. International Journal of Environmental Research and Public Health, Basel, v. 16, n. 17, p. 1-14, 2019. HAAS, Aline Nogueira; GARCIA, Anelise Cristina Dias; BERTOLETTI, Juliana. Imagem corporal e bailarinas profissionais. Revista Brasileira de Medicina do Esporte. Vila Mariana, v. 16, n. 3 (maio/jun. 2010), p. 182-185, 2010.

KAZAREZ, Miguel; VAQUERO-CRISTÓBAL, Raquel; ESPARZA-ROS, Francisco. Percepción y distorsión de la imagen corporal en bailarinas españolas en función del curso académico y de la edad. Nutrición Hospitalaria, Madrid, v. 35, n. 3, p. 661-668, 2018.

KUMAR, Shrawan. Theories of musculoskeletal injury causation. Ergonomics, Reino Unido, v. 44, n. 1, p. 17-47, 2001.

LAPORTA-HERRERO, Isabel et al. Body dissatisfaction in adolescents with eating disorders. Eating and Weight DisordersStudies on Anorexia, Bulimia and Obesity, Londres, v. 23, n. 3, p. 339-347, 2018.

LOPES, André Luiz; DOS SANTOS RIBEIRO, Gustavo. Antropometria aplicada à saúde e ao desempenho esportivo: uma abordagem a partir da metodologia ISAK. Editora Rubio, 2013.

MONTEIRO, Mayara Freitas; CORREA, Márcia Mara. Transtornos alimentares em bailarinas clássicas adolescentes. Revista Brasileira em Promoção da Saúde, Fortaleza, v. 26, n. 3, p. 396403, 2014.

MOURA, Uilla Islany Soares et al. Consumo alimentar, perfil antropométrico e imagem corporal de bailarinas clássicas do Vale 
do São Francisco. Revista Brasileira de Nutrição Esportiva, São Paulo, v. 9, n. 51, p. 237-246, 2015.

MURNEN, Sarah K.; SMOLAK, Linda. The Cash effect: Shaping the research conversation on body image and eating disorders. Body image, Ohio, v. 31, n. 1, p. 288-293, 2019.

NEVES, Clara Mockdece et al. Imagem corporal na infância: uma revisão integrativa da literatura. Rev Paul Pediatr [Internet], São Paulo, v. 35, n. 3, p. 331-9, 2017.

NICHOLS, Tania E. et al. Psychological predictors of body image attitudes and concerns in young children. Body image, Ohio, v. 27, n. 1, p. 10-20, 2018.

NOGUEIRA-DE-ALMEIDA, Carlos Alberto et al. Distorção da autopercepção de imagem corporal em adolescentes. International Journal of Nutrology, Rio de Janeiro, v. 11, n. 2, p. 061-065, 2018. ORGANIZAÇÃO MUNDIAL DE SAÚDE. Global database on Body Mass Index: BMI Classification. 2006. World Health Organization: Geneva, Switzerland, 2015.

PETROSKI, Edio Luiz; PELEGRINI, Andreia; GLANER, Maria Fátima. Motivos e prevalência de insatisfação com a imagem corporal em adolescentes. Ciência \& Saúde Coletiva, Rio de Janeiro, v. 17, n. 4, p. 1071-1077, 2012.

REIS, Nycolle et al. Imagem corporal, estado nutricional e sintomas de transtornos alimentares em bailarinos. Revista Brasileira de Atividade Física \& Saúde, Pelotas, v. 18, n. 6, p. 771-771, 2013.

SCHILDER, Paul. A imagem do corpo: as energias construtivas da psique. 3. ed. São Paulo: Martins Fontes, 1981.

THOMPSON, Joel Kevin. Body image disturbance: assessment and treatment. New York: Pergamon, 233 p. 1990.

VAQUERO-CRISTÓBAL, Raquel; KAZAREZ, Miguel; ESPARZA-ROS, Francisco. Influencia de la modalidad de danza en la distorsión e insatisfacción de la imagen corporal en bailarinas preadolescen- 
tes, adolescentes y jóvenes. Nutricion hospitalaria, Madrid, $v$. 34, n. 5, p. 1442-1447, 2017.

ZIKAN, Fernando Eduardo. Self-reported distortion of body image among classical ballet students in Brazil: classification according to the Body Shape Questionnaire. Fisioterapia Brasil, São Paulo, v. 19, n. 6, p. 821-829, 2019.

\section{Financiamento}

Esse trabalho contou com apoio e financiamento da Universidade Estadual de Goiás (UEG).

\section{Aprovação de comitê de ética em pesquisa}

Pesquisa aprovada pelo Comitê de Ética do Hospital das Clínicas da Universidade Federal de Goiás (UFG). Título: A relação entre os níveis de vitamina $D$ e citocinas inflamatórias com a força muscular e a ocorrência de lesões musculoesqueléticas em bailarino. CAAE: 64312216.8.0000.5078

\section{Publisher}

Universidade Federal de Goiás. Faculdade de Educação Física e Dança. Publicação no Portal de Periódicos UFG. As ideias expressadas neste artigo são de responsabilidade de seus autores, não representando, necessariamente, a opinião dos editores ou da universidade. 